\title{
The Current Dietary Risk Assessment of Chemicals in Food Underestimates the Actual Risk
}

\author{
Jürg A. Zarn* and H. Christoph Geiser
}

\begin{abstract}
Dietary risk assessments (DRA) help determine safe exposure levels of toxic substances in food. Of these, Acceptable Daily Intake (ADI), derived from No Observed Adverse Effect Levels (NOAEL) of longterm toxicity studies, is compared to exposure estimates using lifetime-averaged food intakes. These estimates ignore intermittent high exposures exceeding the ADI; toxic effects of such exposures are considered irrelevant, on the assumption that toxic potency increases with exposure duration, which would be reflected by decreasing NOAELs. However, our statistical analysis of thousands of animal toxicology studies shows that NOAELs after short- and long-term exposure are similar if study design factors are considered. Thus, the short- and long-term potency effects of chemicals are similar. Hence, a short-term toxic effect is generally ignored in current DRA. It accounts for lifetime-averaged but not intermittent high food intakes and, therefore, must be revised. Additionally, there is no added value of long-term studies for ADI derivation.
\end{abstract}

Keywords: ADI $\cdot$ NOAEL $\cdot$ Short-term exposure $\cdot$ Toxicity threshold

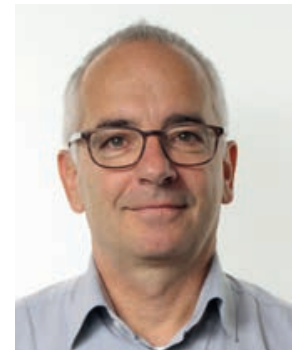

Jürg Zarn obtained his $\mathrm{PhD}$ in biochemistry 1992 at the University of Zürich. From 1992 to 1996 he worked as a scientist at the Laboratory of Oncology at the University Hospital Zürich. Since 1996, he has worked as a pesticide toxicologist at the Swiss Federal Food Safety and Veterinary Office FSVO and became the head of its Toxicology and Biology Sector in 2012. Since 2004, he is member of the Joint FAO/WHO Meeting on Pesticide Residues. His main scientific interests and expertise are in pesticide toxicology and dietary risk assessment.

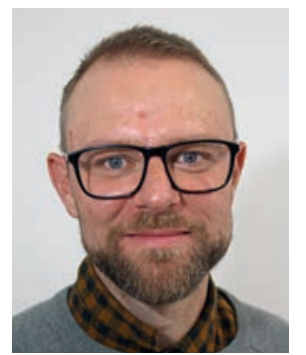

Christoph Geiser obtained his MSc in Cell Biology and Biochemistry in 2003 at the University of Bern, where in 2016 he also completed a postgraduate course in applied statistics. After working at the International Breast Cancer Study Group and the Institute of Forensic Medicine at the University of Basel, he joined the Swiss Federal Food Safety and Veterinary Office FSVO in 2013 where he has since worked as a pesticide toxicologist. Since 2018, he is advising the FAO/WHO Joint Meeting on Pesticide Specifications on toxicological matters. His main scientific interests and expertise are in pesticide toxicology and dietary risk assessment.

\section{Background}

The dietary risk assessment (DRA) is used to determine whether the exposure levels of contaminants and pesticide residues in food are safe for human consumption. The DRA compares exposure levels with health-based guidance values (HBGVs), which are derived from No Observed Adverse Effect Levels (NOAEL) identified in animal toxicity studies. The NOAEL is the highest non-toxic dose, the next higher dose is called the Lowest Observed Adverse Effect Level (LOAEL). The HBGV is obtained by dividing the numerical value of NOAEL by a safety factor, which represents the variability of sensitivity within the human population, differences between the tested animal models and humans, and, when applicable, the uncertainty in the available database. It is thus assumed that no human subpopulation is more sensitive than an animal model by a factor greater than the safety factor. The designation of HBGV may differ in different parts of the world; in Europe, for example, the HBGV for chronic toxicity in contaminants is expressed as Tolerable Daily Intake (TDI) and pesticide residues as Acceptable Daily Intake (ADI). The values themselves are derived in a similar fashion and represent the dose of a specific substance that can be ingested on a daily basis without any health risk. Conceptually, ADI/TDIs in humans correspond to NOAELs in animals.

\section{Toxicity Threshold and Exposure Duration}

A more or less explicit assumption in regulatory toxicology is that toxicity correlates with exposure duration. Therefore, occasional exposures exceeding the ADI are regarded as toxicologically non-relevant. Only if exposures continually exceed the ADI, are measures taken to reduce the exposure levels below the ADI. However, there is little specification regarding the exact aspect of toxicity that is meant to correlate with exposure duration, the number of qualitatively different effects, or severity of effects at a given dose, or the toxicity threshold approximately by the values of the NOAELs and LOAELs. It has been reported that the number of exposures and nature and/or severity of effects correlate with exposure duration. Furthermore, slightly lower NOAEL and LOAEL values are usually observed when the exposure duration of a substance is increased. This suggests that the toxic potencies of substances increase with increasing exposure durations. However, this would be in conflict with the paradigm of regulatory risk assessment that for all compounds, except DNA-reactive compounds, thresholds of 
toxicity exist and exposures below toxicity thresholds are non-toxic. The NOAEL and LOAEL represent the lower and the upper boundaries of the experimental approximation of the toxicity threshold. A decrease in NOAELs with increased exposure durations would suggest that thresholds of toxicity are age-dependent, where older subjects are more sensitive to toxicity than their younger counterparts. This is contrary to the general assumption that young animals are more susceptible than old animals. Furthermore, a decrease in NOAEL with increasing exposure duration would imply that there could be no physiological changes in tested animals on one day but toxic responses on the other day. Such an observation would violate the concept of the thresholds of toxicity. The paradigm of the threshold of toxicity also implies that if after long-term exposure, a toxic effect at a given dose is observed, then precursor changes indicative of the toxic effect must have occurred before the observation was noted. These precursor effects must have existed before the apical toxic effect was first observed, since the given dose exceeded the toxicity threshold.

In conclusion, although even a single exposure above the toxicity threshold approximated by the NOAEL may induce adverse changes, repeated exposures are safe, as long as they are below the threshold of toxicity. Arguing to the contrary would imply that either toxicity thresholds do not exist or are subject to change without the occurrence of changes in any parameter prior to the manifestation of an apical toxic effect.

To address the contradiction between the paradigm of toxicity thresholds and the slight decrease of NOAELs with exposure duration increase in animal toxicity studies, we analysed several thousands of toxicological studies of different durations on more than 400 pesticides in order to elucidate the dependence of NOAELs and LOAELs on exposure duration. ${ }^{[1]}$

\section{NOAELs are Independent of Exposure Duration}

Previous studies have examined the relationship between NOAELs and exposure duration, and the relevant literature was recently reviewed. ${ }^{[1,2]}$ In these studies, a wide variety of chemical groups was represented. Most of these evaluations have shown that shorter-term exposure studies have slightly higher NOAELs than longer-term exposure studies. However, the average differences between short- and long-term exposure NOAELs for all chemical groups were well within a factor of 10 and were similar between chemical groups. The observed small difference was not dependent on group chemical structure and thus not related to biochemical interactions, i.e. toxicity. We hypothesized that this minor, yet consistent, chemical-independent difference between shorter-term and longer-term NOAELs for given chemicals is not due to toxicological reasons. To test our hypothesis, we performed statistical analyses on a wide range of regulatory animal toxicity studies, involving mice, rats, and dogs, and different exposure durations conducted on pesticide compounds widely varying in structure. ${ }^{[1-3]}$ We were able to identify the number of animals per group, dose spacing, and the decrements ${ }^{[4]}$ of the actual substance intake with increasing duration, but not exposure duration, as the explanatory variables for the observed small differences between short-term and longterm NOAELs. When adjusting for these explanatory variables, which represent study design factors, no statistically significant differences between NOAELs from studies ranging from 2 to 104 weeks of exposure was observed. We thus demonstrated that the toxic potency is comparable after short-term and long-term exposure, and this holds true independent of the animal species and the chemical structure tested. The data actually verify the paradigm of toxicity thresholds that any exposure above the NOAEL may be adverse and even repeated long-term exposure below the NOAEL is safe.

There are three exceptions in which the NOAELs may actually differ depending on exposure duration. First, foetuses or young adult animals could be more sensitive than older animals. However, this would result in lower short-term NOAELs compared to long-term NOAELs, which is contrary to the tacit assumption that toxicity increases with an increase in exposure duration. Second, the threshold of toxicity can be age related, i.e. older subjects have a lower threshold for toxicity than their younger counterparts, which would result in higher short-term NOAELs compared to long-term NOAELs. Third, certain compounds, such as polychlorinated biphenyls (PCB), accumulate in the body. Continued exposure with low doses of accumulating compounds may lead to a concentration build-up in the body and after a certain duration of exposure, the toxicity threshold may be exceeded and toxicity will consequently occur.

\section{Implications for Regulatory Chemical Dietary Risk Assessment}

\subsection{The ADI Applies for Every Exposure Duration}

As our analysis has shown, the fact that the toxicity thresholds of most compounds are fixed and independent of exposure duration divides the exposure range into two categories: safe and unsafe. The maximum exposure range allowed by the NOAEL is always safe regardless of exposure duration, whereas exposures above that maximum range of the NOAEL are possibly toxic even if the exposure is only short-term. Consequently, the ADI must not be viewed as a 'chronic' HBGV but rather as a generally applicable HBGV. Exposures should never exceed the ADI.

\subsection{Long-term Studies for Dietary Risk Assessment Provide no Added Value}

An exposure exceeding the NOAEL will result in toxicity, prolonging the exposure may increase the severity of the toxicity, which may ultimately become irreversible and, thus, hinder possible recovery. Clearly, to determine all hazardous properties of a compound, the exposure duration should be maximized. However, for the purpose of hazard characterization (dose-response considerations and identification of toxicity thresholds), approximation of the toxicity threshold with the NOAEL of a robust, well-designed, high-powered short-term study is sufficient to derive an ADI for use in risk assessment. Conversely, performing a longterm study to identify the NOAEL approximating the toxicity threshold obviously has no added value over well-conducted high powered short-term studies.

\subsection{Reconsidering the Exposure Assessment}

In the DRA, consumer exposure is essentially estimated by multiplying the concentration of a particular compound with the amount of the respective food commodity consumed. The DRA of contaminants differs from the DRA of pesticide residues, since the former allows for flexibility, whereas the latter is defined by quite rigid procedures. ${ }^{[4]}$ In a chronic DRA of pesticide residues, food consumption values are derived from food balances, which represent the difference between a country's production (including importation) minus exportation. This value then is divided by the country's entire population to calculate the daily consumption per capita. This calculation results in values of chronic daily food intakes being often much lower than the actual food intakes. Using these very low food consumption values suggests that a certain pesticide residue level is acceptable because the purely arithmetically calculated exposure value is below the ADI (Fig. 1). However, the fact that intermittent exposures on the actual food consumption exceed the ADI may be concealed..$^{[1]}$

Our study suggests that any single exposure above the NOAEL in animals and, hence, above the ADI in humans may result in toxic effects. Therefore, a DRA revision is necessary to take into account the short-term toxic potency of compounds. Any exposures exceeding the ADI should be identified and managed. Some 


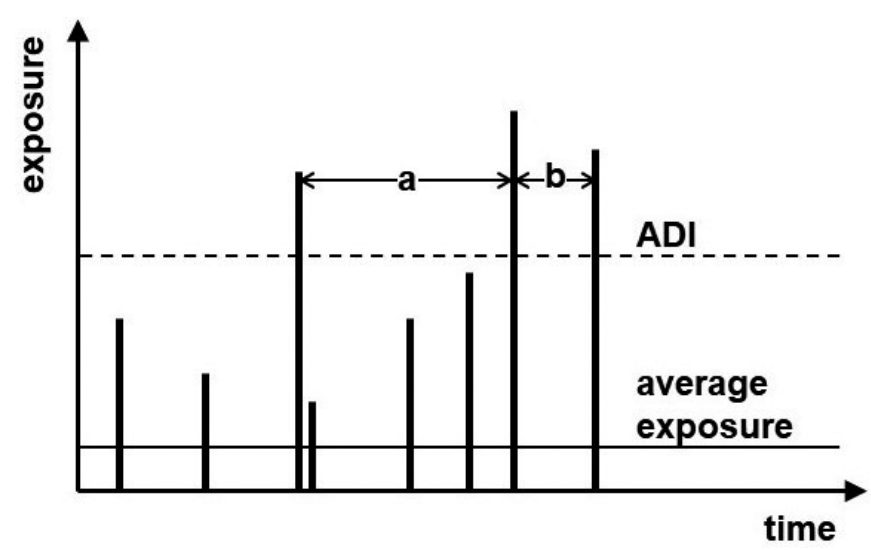

Fig. 1. Intermittent exposures above the ADI. Bars represent exposures to a chemical in food. The solid horizontal line represents the average daily intake of such a chemical whereas the dashed line represents the $\mathrm{ADI}$. According to the current dietary risk assessment, the figure shows no public safety concern since the average exposure is below the ADI. However, the short-term toxic effect of chemicals is ignored. The frequency of a chemical exposure that exceeds the ADI must also be considered. For example, in the figure, if period a between the two exposures exceeding the ADI was long enough to allow for full recovery from all biological changes caused by the exposures, then the two exposures are considered toxicologically independent and therefore acceptable. By contrast, if the recovery period $b$ was too short for full recovery, then the two exposures above the ADI separated by period b may not be acceptable, as permanent adverse effects may be induced.

initiatives to revise the DRA to also consider the short-term toxic effects of chemicals have been launched. ${ }^{[5]}$

\section{Proposal for Amendments in Chemical Dietary Risk Assessment}

DRAs of compounds should use actual high food consumption to identify exposures exceeding the ADI that could occur intermittently in real life situations. Regarding pesticides, the International Estimated Short-Term Intake (IESTI) model provides a tool to identify realistic high exposures resulting from the consumption of a single meal or from meals consumed in a single day. ${ }^{[6]}$ The IESTI model could also be used to determine the exposure levels of substances other than pesticide residues. Along with identifying any exposures exceeding the ADI, there are two other variables that are important to identify, namely, the frequency of such exposures and the duration of exposure below ADI which represents the recovery phase (Fig. 1). Identifying the mechanism behind the observed toxic effects is essential to understand the physiological consequence of the recurring exposures exceeding the ADI and to determine the minimal period of exposure below the ADI needed to enable full recovery from the effects induced by exposure above the ADI. If the mechanism of toxicity for a compound is not fully understood, any exposure that exceeds the ADI should be considered adverse.

\section{Conclusion}

The finding that NOAELs are independent of exposure duration and toxic effects caused by a compound even after a shortterm exposure calls for a revision of the current DRA, to prevent any exposure exceeding the ADI. The IESTI exposure model provides an accurate estimate of the daily intake of a compound. It is critical to identify and evaluate any possible exposures that could exceed the ADI to ensure public health safety. It is safe to assume that an exposure exceeding the ADI regardless of the frequency of exposure may be hazardous to public health.

\section{Abbreviations}

ADI: Acceptable Daily Intake

DRA: Dietary Risk Assessment

HBGVs: Health-Based Guidance Values

IESTI: International Estimated Short-Term Intake

LOAEL: Lowest Observed Adverse Effect Level

NOAEL: No Observed Adverse Effect Levels

TDI: Tolerable Daily Intake

Received: July 11, 2019

[1] J. A. Zarn, C. D. O'Brien, Arch. Toxicol. 2018, 92, 157, DOI: 10.1007/ s00204-017-2052-4

[2] J. A. Zarn, B. E. Engeli, J. R. Schlatter, Regul. Toxicol. Pharmacol 2011, 61, 243, DOI: 10.1016/j.yrtph.2011.08.004.

[3] J. A. Zarn, E. Hanggi, A. Kuchen, J. R. Schlatter, Regul. Toxicol. Pharmacol. 2010, 58, 72, DOI: $10.1016 /$ j.yrtph.2010.04.007.

[4] J. A. Zarn, B. E. Engeli, J. R. Schlatter, Regul. Toxicol. Pharmacol 2013, 67, 215, DOI: $10.1016 /$ j.yrtph.2013.07.012.

[5] D. Arcella, A. Boobis, P. Cressey, H. Erdely, V. Fattori, J. C. Leblanc, M. Lipp, R. Reuss, S. Scheid, A. Tritscher, T. Van der Velde-Koerts, P. Verger, Crit. Rev. Toxicol. 2019, 49, 1, DOI: 10.1080/10408444.2019.1578729

[6] a) EFSA, PRIMo - Pesticide Residue Intake Model, https://www.efsa.europa.eu/de/applications/pesticides/tools, accessed 6 February, 2019; b) WHO, Global Environment Monitoring System (GEMS/food), http://www.who.int/ foodsafety/areas_work/chemical-risks/gems-food/en/, accessed 6 February, 2019. 